\title{
Erratum to: Halolamina rubra sp. nov., a haloarchaeon isolated from non-purified solar salt
}

\author{
In-Tae Cha $\cdot$ Kyung June Yim $\cdot$ Hye Seon Song $\cdot$ Hae-Won Lee \\ Dong-Wook Hyun · Kil-Nam Kim • Jong-Soon Choi • Daekyung Kim • \\ Sung-Jae Lee $\cdot$ Myung-Ji Seo $\cdot$ Hak-Jong Choi $\cdot$ Jin-Woo Bae $\cdot$ \\ Sung-Keun Rhee $\cdot$ Seong Woon Roh $\cdot$ Young-Do Nam
}

Published online: 26 June 2015

(C) Springer International Publishing Switzerland 2015

\section{Erratum to: Antonie van Leeuwenhoek (2014) \\ 105:907-914 \\ DOI 10.1007/s10482-014-0145-0}

In the original publication of the article, the Electronic Supplementary figures 1 and 2 were omitted inadvertently. The missing ESM figures are provided in the link below.

The online version of the original article can be found under doi:10.1007/s10482-014-0145-0.

Electronic supplementary material The online version of this article (doi:10.1007/s10482-015-0507-2) contains supplementary material, which is available to authorized users.

I.-T. Cha · K. J. Yim · H. S. Song · H.-W. Lee

K.-N. Kim · D. Kim · S. W. Roh $(\bowtie)$

Jeju Center, Korea Basic Science Institute, Jeju 690-140, Republic of Korea

e-mail: seong18@gmail.com

I.-T. Cha · S.-K. Rhee

Department of Microbiology, Chungbuk National

University, Cheongju 361-763, Republic of Korea

H.-W. Lee · H.-J. Choi

World Institute of Kimchi, Gwangju 503-360, Republic of Korea

D.-W. Hyun · S.-J. Lee · J.-W. Bae

Department of Life and Nanopharmaceutical Sciences and Department of Biology, Kyung Hee University,

Seoul 130-701, Republic of Korea
J.-S. Choi

Division of Life Science, Korea Basic Science Institute, Daejeon 305-806, Republic of Korea

\section{J.-S. Choi}

Graduate School of Analytical Science and Technology, Chungnam National University, Daejeon 305-764, Republic of Korea

\section{M.-J. Seo}

Division of Bioengineering, Incheon National University, Incheon 406-772, Republic of Korea

\section{Y.-D. Nam ( $\square)$}

Fermentation and Functionality Research Group, Korea Food Research Institute, Sungnam 463-746, Republic of Korea

e-mail: youngdo98@kfri.re.kr 